\title{
Unidirectional stripping extraction from a cyclotron which accelerates light as well as heavy ions
}

\author{
Jasna L. Ristić-Djurović and Saša Ćirković \\ Vinča Institute for Nuclear Sciences, P.O. Box 522, 11001 Belgrade, Serbia
}

(Received 2 December 2002; published 12 March 2003)

\begin{abstract}
The VINCY Cyclotron (VINča CYclotron) is a multipurpose machine intended to accelerate light as well as heavy ions. To extract heavy ions with low energy and light ions an extraction system with stripping foil is used. Heavy ions with high energy will be extracted by means of an electrostatic deflector. The former extraction system will be manufactured and used first. The proposed unidirectional stripping extraction system is the optimal balance between the placement of the extraction line and the required diversity and quality of the extracted beam. The available range of extraction directions is set by geometry limitations.
\end{abstract}

DOI: 10.1103/PhysRevSTAB.6.033501

PACS numbers: 29.27.Ac, 29.20.Hm

\section{INTRODUCTION}

The TESLA Accelerator Installation, which is under construction in the Vinča Institute for Nuclear Sciences, consists of three machines: the VINCY Cyclotron as the main machine, the pVINIS Ion Source which is a volume light ion source, and the mVINIS Ion Source which is an electron cyclotron resonance heavy ion source. The TESLA Accelerator Installation is intended for commercial as well as for experimental applications. The first programs of use of the VINCY Cyclotron are related to routine and experimental production of radioisotopes and radiopharmaceuticals and to biomedical research. The project had a pending status from 1998 until recently. During the fall of 2002 the partial funding granted by domestic as well as foreign institutions has reopened the project. To meet the obtained investments, the project has been reorganized into phases and additional investors for later phases are being searched for. The completion of the first, so-called fast track, phase is scheduled for June 2005 [1].

The VINCY Cyclotron is a compact four-sector isochronous cyclotron. The bending and focusing constants of the machine are 145 and $75 \mathrm{MeV}$, respectively, and its pole diameter is $2 \mathrm{~m}$. The radio frequency system consists of two $\lambda / 4$ resonators with eigenfrequency in the range from 17 to $31 \mathrm{MHz}$. The phase difference between the resonators can be $0^{\circ}$ as well as $180^{\circ}$. The operating diagram of the VINCY Cyclotron and operating limitations introduced by the magnetic structure and rf system of the machine are shown in Fig. 1 [2]. The first beam will be extracted from the machine using the ion stripping method.

During interaction with a thin carbon foil some of an ion's electrons are stripped and thus the rotation radius of a positive ion is decreased or, in the case of a negative ion, the rotation direction is changed. An ion is defined as positive or negative according to its charge during acceleration. The ion stripping method is very efficient in extracting light ions, for example, $\mathrm{H}^{-},{ }^{2} \mathrm{H}^{-}, \mathrm{H}_{2}{ }^{+}, \mathrm{He}^{+}$, represented in the operating diagram of the VINCY Cyclotron by the curves $\eta=1,0.5,0.5$, and 0.25 , respectively. Stripping extraction of heavy ions is less efficient, since it is limited to only one of the charge states among which the beam is distributed after interaction with the foil. After stripping, positive ions make at least one loop before they leave the cyclotron. In order to avoid disturbances caused by the cyclotron magnetic and electric fields that are not designed for loop motion, it is advisable to consider only single-loop beams that do not interact with the acceleration gaps after stripping. To achieve this, the radius of a loop must be sufficiently small and, consequently, the ratio of ion specific charges after and before stripping has to be larger than roughly 2 . Looking at the operating diagram, stripping extraction of heavy ions is therefore restricted to ions with $\eta \leq 0.25$. The ion beams that cannot be extracted by stripping will be extracted from the VINCY Cyclotron by an electrostatic deflector.

\section{CONDITIONS AND ASSUMPTIONS}

The placement and acceptances of the high-energy transport line have been preset. The transport line begins at the point whose coordinates are $R=3 \mathrm{~m}$ and $\theta=-15^{\circ}$, while its direction is defined with $\varphi=$ $-24.25^{\circ}$, as depicted in Fig. 2. At $R=3 \mathrm{~m}$, the transport line acceptances in both the horizontal and vertical directions satisfy $a<25 \mathrm{~mm}$ and $P_{a}<10 \mathrm{mrad}$, where $a$ and $P_{a}$ are spatial and momentum coordinates of an ion with respect to the transport line axis, respectively.

The horizontal cross section of the VINCY Cyclotron's vacuum chamber is octagonal and is painted white in Fig. 2. The size of the doorway on the vacuum chamber side, designated for beam extraction, sets the angular width of the space available for extraction system elements to $\theta \in\left[-16^{\circ}, 16^{\circ}\right]$. The vertical gap between sectors of $31 \mathrm{~mm}$ is rather small and this complicates the mechanical design of the stripping foil holder and may limit the area of stripping foil placement. 


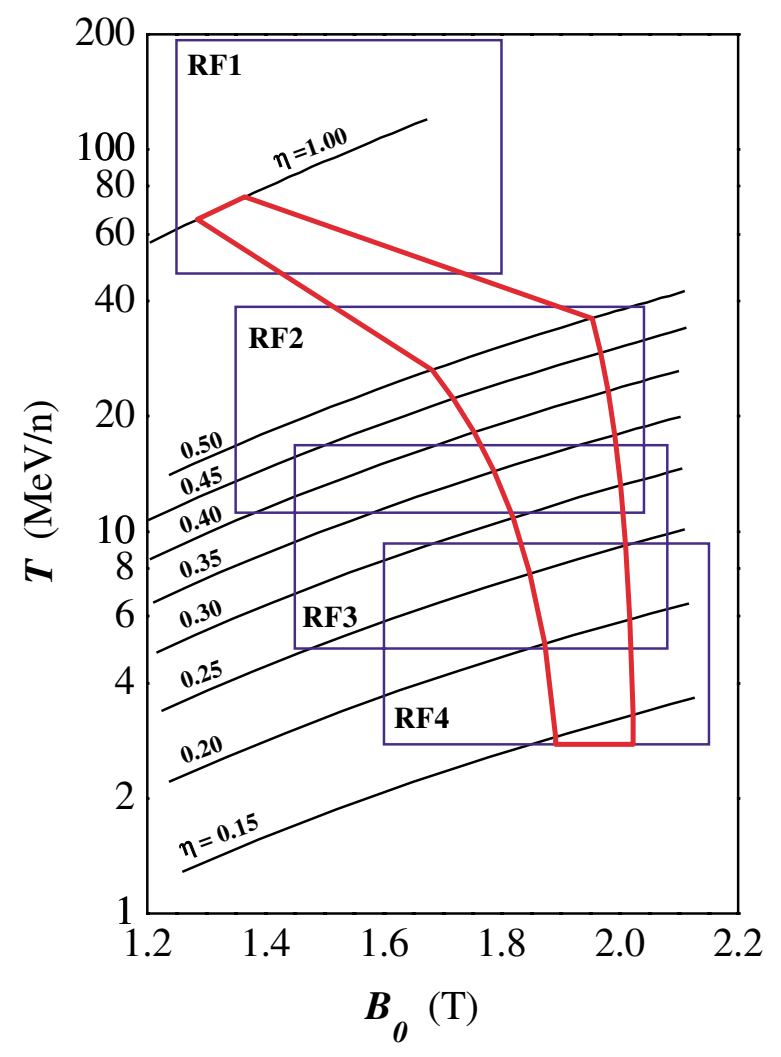

FIG. 1. (Color) Operating diagram of the VINCY Cyclotron. The dependence of the desired ion energy per nucleon, at the extraction radius of $0.86 \mathrm{~m}, T$, on the needed magnetic field in the machine center, $B_{0}$, is shown. The specific charge of an ion, $\eta$, is used as a curve parameter. The calculations are performed using Gordon's procedure and the measured magnetic field maps. The horizontal sides of the four rectangles define the lower and upper limits of the rotation frequency, i.e., extraction energy, of an ion accelerated using the first, second, third, or fourth harmonic modes of the rf system. The left border of the operating range is set by the trim coils capability to account for the maximum of $450 \mathrm{mT}$ difference between the isochronous field and the field obtained by the main coils only. For $\eta \leq 0.5$ the right border corresponds to the maximum main coils current of $1000 \mathrm{~A}$, while for $\eta=1$ the largest obtainable energy is set by the focusing capabilities of the machine.

The elements of the extraction system are designed via computer simulation of beam dynamics, using a self-developed software package [3]. The isochronized magnetic field needed for acceleration of a particular ion is calculated taking into account the measured magnetic field maps of the VINCY Cyclotron [4]. It was assumed that the stripping foil is positioned along an equilibrium orbit, which is defined by its mean radius, $R_{m}$. For the ions with $\eta=1$, right after interaction with the foil, the values of beam emittances in the horizontal and vertical directions are taken to be $\varepsilon_{h}=$ $1 \mathrm{~mm} 5 \mathrm{mrad} \pi=5 \pi \mathrm{mm} \mathrm{mrad}$ and $\varepsilon_{z}=5 \mathrm{~mm} 1 \mathrm{mrad}$ $\pi=5 \pi \mathrm{mm}$ mrad, respectively. For all other ions these values are assumed to be $\varepsilon_{h}=2 \mathrm{~mm} 6 \mathrm{mrad} \pi=$

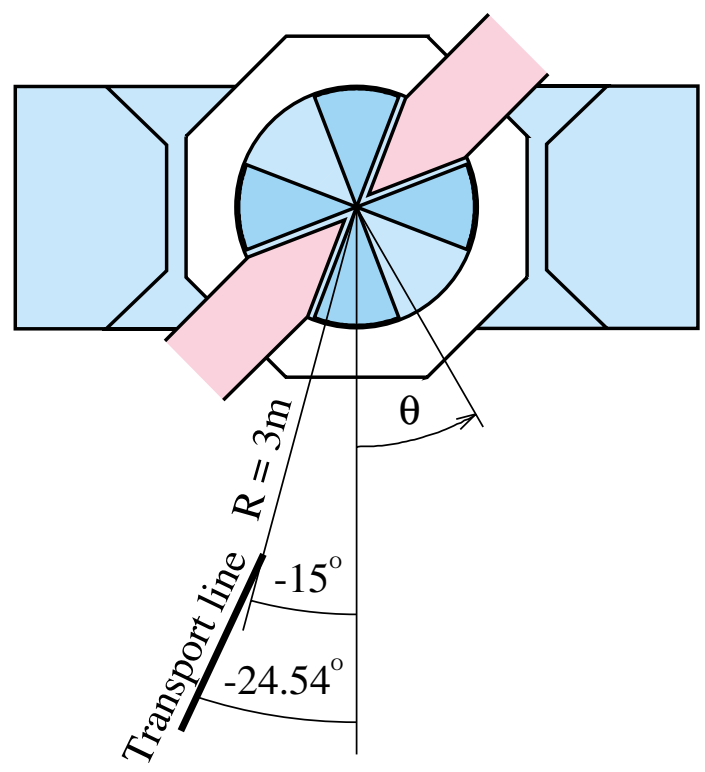

FIG. 2. (Color) Schematics of the VINCY Cyclotron. The median plane cross sections of the yoke, pillars, pole (light blue), vacuum chamber (white), and rf resonators (pink) are shown. The azimuthal coordinate system used further throughout the paper is defined by its referent zero-angle axis and the positive $\theta$ direction. Also shown are the required direction and placement of the high-energy transport line.

$12 \pi \mathrm{mm} \mathrm{mrad}$ and $\varepsilon_{z}=5 \mathrm{~mm} 1 \mathrm{mrad} \pi=5 \pi \mathrm{mm}$ mrad. The energy spread of an ion beam at the foil is taken to be $\pm 1 \%$.

The stripping extraction system should cover as much as possible of the operating diagram of the machine, however, it is required that special attention is paid to the two cases. These are the protons obtained by stripping from $\mathrm{H}^{-}$and the protons obtained from $\mathrm{H}_{2}{ }^{+}$. In addition, the energy decrease of the protons obtained from $\mathrm{H}_{2}{ }^{+}$is to be obtained without changing the operating regime of the machine. This is achieved by adjusting the foil position to lower $R_{m}$. The desired energy span of the protons obtained from $\mathrm{H}_{2}{ }^{+}$ranges from 30 to $\sim 19 \mathrm{MeV}$.

\section{DESIGN}

The stripping extraction system consists of the stripping foil, mechanism for foil positioning and exchange, and beam-guiding elements positioned between the stripping foil and the high-energy transport line. During the design of the stripping extraction system, a beam dynamics simulation is used as a tool in the process of investigating and defining parameters of all the system elements. The design of the system is completed when the optimal set of system parameters is obtained. However, each element of the system is yet to be designed and, for this design, the parameters from the optimal set of system parameters corresponding to a particular element are used as requirements. The following system 
parameters are defined during the system design process: the operating area of the stripping foil, the placement, dimensions, and focusing and/or bending characteristics of the beam-guiding elements. To enable directing of all desired beams into the high-energy transport line, the beam-guiding elements used for focusing and bending of a beam are chosen to be active magnetic elements positioned outside the vacuum chamber. The positioning of the beam-guiding elements is restricted to $R \geq 1.6 \mathrm{~m}$ by the vacuum chamber dimensions. The radial position of a beam exit point from the cyclotron is therefore set as $R_{e}=1.6 \mathrm{~m}$. Consequently, the beam exit point is defined only by its azimuthal position, $\theta_{e}$. It can be shown that performance of the system, as well as the system design parameters, depends on the point of a beam's exit from the cyclotron [5]. Therefore, $\theta_{e}$ is used as the key design parameter as well as an argument in graphical representation of system performances.
If a cyclotron magnet is saturated, it can be proved that ions can be divided into classes in such a way that all ions within a given class behave the same in terms of stripping extraction [5]. Each class is defined by the corresponding values of $R_{m}$ and $K$, where $R_{m}$ is the mean radius of an equilibrium orbit, as defined earlier, and $K$ is the ratio between the specific charges of an ion after and before stripping. Within a given class, the extraction energy of an ion depends on the ion's specific charge before stripping. Figure 3 gives the dependence of the position of the beam exit point on the foil position for various classes of ions. These dependencies and the results presented in Ref. [5] illustrate how beam diversity and quality are influenced by the choice of the key design parameter, $\theta_{e}$. Note that the protons obtained from $\mathrm{H}^{-}$ions have $K=-1$, whereas the protons obtained from $\mathrm{H}_{2}{ }^{+}$ions belong to the ion class with $K=2$.

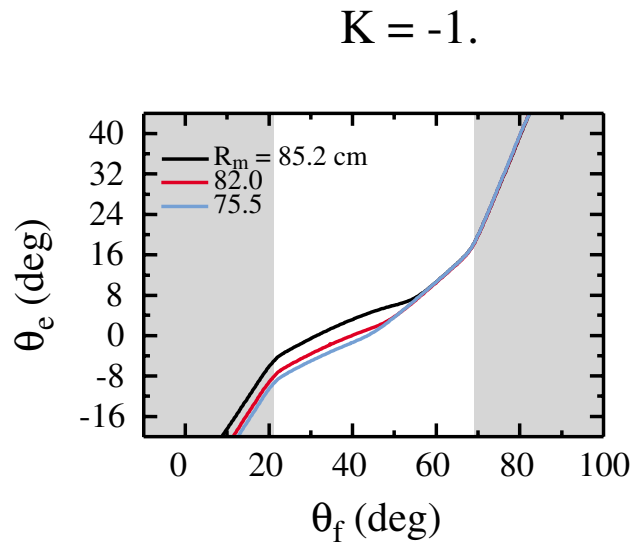

$\mathrm{K}=2.5$

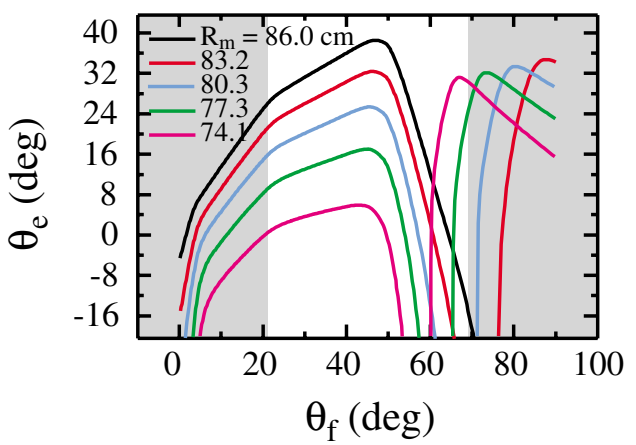

$\mathrm{K}=2$.

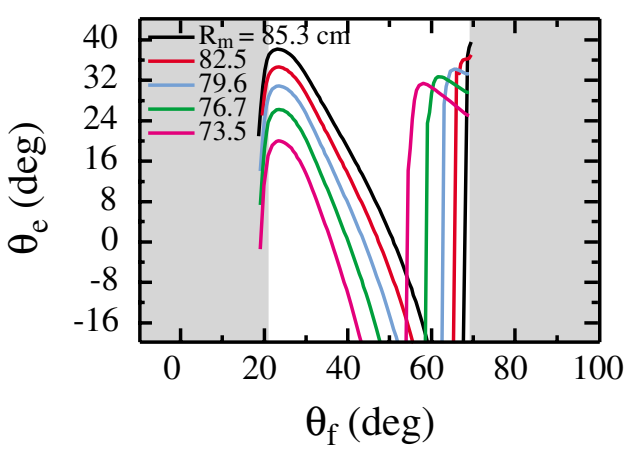

$$
\mathrm{K}=3 \text {. }
$$

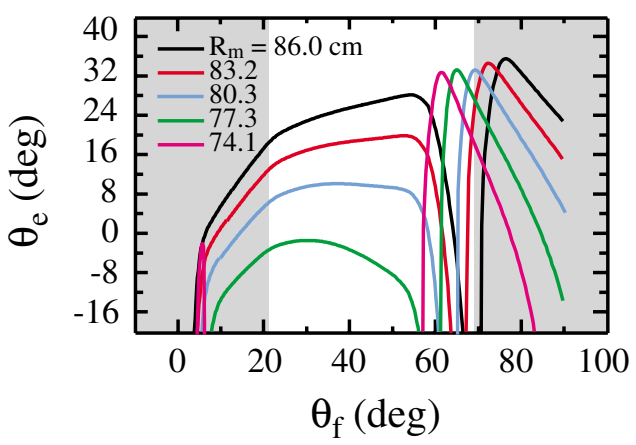

FIG. 3. (Color) Exit point dependence on foil position. A beam exit point from the cyclotron as well as a foil position is fully determined by their azimuthal coordinates, $\theta_{e}$ and $\theta_{f}$, respectively. This is because the radial position of an exit point is $R_{e}=$ $1.6 \mathrm{~m}$, as limited by the vacuum chamber size, while the foil is positioned along an equilibrium orbit defined by the orbit mean radius $R_{m}$. The shaded areas depict the sector placement. The curve discontinuities in the graphs corresponding to positive ions $(K>0)$ are caused by sector edge gradients and they mark transition from a single-loop to a double-loop ion trajectory after the foil. 
After stripping, a negative ion changes its rotation direction and exits the cyclotron. Consequently, the azimuthal coordinate of the exit point increases with the azimuthal coordinate of the foil position, as illustrated in Fig. 3, by the graph corresponding to $K=-1$. A positive ion does not change rotation direction; instead, its rotation radius is decreased and before it leaves the cyclotron it executes at least one loop. Positive ion beam dynamics is therefore heavily influenced by the sector edge gradients [5]. Since beam trajectories that perform more than one loop after the foil are characterized by beam losses, only a single-loop extraction is considered. In Fig. 3, the parts of the curves corresponding to a single-loop extraction are placed to the left of the discontinuities, that is, at lower $\theta_{f}$ 's.

On the graphs in Fig. 3, a straight line would represent the chosen value of $\theta_{e}$. The intersection between this line and the $\theta_{e}\left(\theta_{f}\right)$ curves defines the foil positions that will bring a beam into the chosen exit point. For $K>0$ and high enough values of $R_{m}$ one can choose between two available foil positions. For the limiting case, the line corresponding to the chosen value of $\theta_{e}$ is tangent to the $\theta_{e}\left(\theta_{f}\right)$ curve corresponding to the smallest obtainable $R_{m}$, and only one suitable foil position is available. Therefore, for $K>0$, the chosen exit point position sets the minimum $R_{m}$, i.e., beam energy, obtainable through the single-loop extraction. As stated earlier, the exit point position is restricted to the region $\theta_{e} \in\left[-16^{\circ},+16^{\circ}\right]$.

Beam quality, measured by beam emittances and envelopes is deteriorated when a beam passes close to a sector edge. For $K>2$, if a loop after the foil is placed away from the influence of sector edge gradients, the quality of the extracted beams does not vary much with $\theta_{f}$. These regions of good quality beams correspond to the lowslope regions of the single-loop portions of $\theta_{e}\left(\theta_{f}\right)$ curves [6]. Consequently, for any value of $\theta_{e}$, the energy range of good quality beams is relatively narrow, if the energy of a beam is to be changed by the foil position adjustment. Difficulties related to the mechanical design of the foil holder and exchange mechanism may cause the foil positions over sectors to be unachievable. The remaining backup foil position in the valley provides beams of poorer quality.

For the beams with $K \cong 2$ and $K<0$, the value of $\theta_{e}$ does not influence beam quality significantly. For $K=$ -1 beam quality is not significantly disturbed since a beam exits the cyclotron without performing a loop after the foil, while for $K \cong 2$ negative influence of sector edge gradients on beam quality cannot be avoided due to the loop size.

One of the two types of beams that are of special importance has $K=2$ and the disturbed quality of this beam must be attended and improved promptly. The focusing element should decrease the radial envelope of the beam as soon as possible, therefore it should be positioned at the exit point. Also, the axis of the element should follow the beam direction, if the focusing function of the element is to be fully used. The last condition is satisfied if the exit point is at $\theta_{e} \cong 6^{\circ}$, as can be seen in Fig. 4. The required bending properties of the extraction line elements are proportional to $\Delta \varphi$, so a beam does not need to be bent if $\Delta \varphi=0$. For $K=2$, the dependence $\Delta \varphi\left(\theta_{e}\right)$ does not vary much with beam energy, i.e., $R_{m}$, and the focusing function of the element positioned in the exit point $\theta_{e}=6^{\circ}$ can be used efficiently for all the beams from the required energy range.

The requirements for beam bending are minimal if the extraction is two directional, that is, if the extraction line has two branches with the exit points $\theta_{e 1} \cong 6^{\circ}$ and $\theta_{e 2} \cong$ $-7.5^{\circ}$ for beams with $K>0$ and $K<0$, respectively. The distance between the two exit points is $\sim 38 \mathrm{~cm}$, which does not leave enough room for two extraction tubes and a "picture frame" shape of the focusing magnet. Therefore, both extraction branches have to be within the same extraction tube. The two beam-guiding elements positioned along the extraction branch corresponding to $\theta_{e 1}$, therefore, must have a "C" shape. The two elements in question are the focusing element and the bending element needed for beams with $K \neq 2$. The size of the extraction tube for the two-directional extraction does not leave much space for the stripping foil holder and exchange mechanism, and vacuum pumps. The additional bending magnet (BM) must be positioned at the beginning of the transport line and used for the adjustment of the extracted beam directions to the high-energy transport line direction.

The unidirectional stripping extraction system extracts all the beams through a single exit point, the point with $\theta_{e}=6^{\circ}$, defined earlier as the optimal for the higher priority proton extraction from $\mathrm{H}_{2}{ }^{+}$ions. The difference between the direction of the extraction line

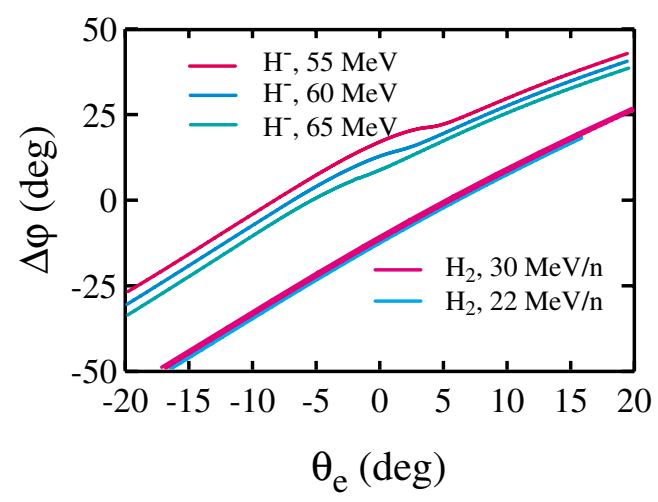

FIG. 4. (Color) Bending angle dependence on the exit point. A required bending angle, $\Delta \varphi$, is defined as an angular difference between a beam direction and an extraction line direction. The extraction line connects the exit point and the starting point of the transport line. The dependencies $\Delta \varphi\left(\theta_{e}\right)$ are given for the classes of beams with $K=-1$ and $K=2$, to which belong the two beams with higher priority. 
corresponding to this exit point and the direction of the remaining higher priority beams of protons obtained from $\mathrm{H}^{-}$is of the order of $20^{\circ}$; see Fig. 4. The large value of $\Delta \varphi$ requires that the magnetic element, positioned at the exit point, has a bending function in addition to the focusing function required for the $K=2$ case. It was assumed that the magnetic field inside the element with the combined focusing and bending function is $\boldsymbol{B}_{\mathrm{CM}}=$ $G \cdot z \cdot \boldsymbol{h}+\left(B_{\mathrm{CM}}+G \cdot h\right) \cdot \boldsymbol{z}$, where $\boldsymbol{h}$ and $h$, and, $z$ and $z$ are unit vectors and coordinates, in the horizontal and vertical directions, respectively. Directions are determined with respect to the axis of the combined magnet (CM). $G$ and $B_{\mathrm{CM}}$ are the combined magnet parameters in $\mathrm{T} / \mathrm{m}$ and $\mathrm{T}$, respectively, and, for each ion beam, their values are determined through a computer simulation of beam dynamics. For the beams with $K>0$ the bending function of the element with a combined focusing and bending function is used for slight adjustments of a beam direction to the extraction line direction. If $K>0$, the predominant function of the combined element is beam focusing in the radial direction. For the beams with $K<0$, only the bending function of the combined element is needed. Since the extraction tube contains a single extraction branch, beam envelopes limit the width of the extraction tube and the aperture of the combined element. However, the combined element is not limited to a $\mathrm{C}$ shape and can have a picture frame shape. As was the case with the two-directional extraction, a bending magnet is positioned in the initial point of the high-energy transport line and used to change beam direction from the extraction line direction to the high-energy transport line direction. In beam dynamics simulations, it was assumed that the magnetic field inside the bending magnet is $\boldsymbol{B}_{\mathrm{BM}}=B_{\mathrm{BM}} \cdot \boldsymbol{z}$, where $\boldsymbol{z}$ is a unit vector in the vertical direction and $B_{\mathrm{BM}}$ is the magnet parameter whose value is to be determined for each ion beam. Beam dynamics simulations were performed assuming that the effective lengths of the combined and bending magnets are $L_{\mathrm{CM}}=70 \mathrm{~cm}$ and $L_{\mathrm{BM}}=60 \mathrm{~cm}$, respectively.

The unidirectional stripping extraction system has a number of advantages compared to the two-directional stripping extraction system. The unidirectional system has only two beam-guiding elements compared to three such elements needed for the two-directional extraction. The extraction tube of the unidirectional system leaves much more space for the foil positioning and exchange mechanism and vacuum pumps. The two beam-guiding elements of the unidirectional system, namely, the combined element and the bending magnet, can both have a picture frame shape, and consequently better bending and focusing properties compared to the properties of the beam-guiding elements of the two-directional system, restricted to a $\mathrm{C}$ shape. To extract protons from the $\mathrm{H}^{-}$ beam the stripping foil is positioned over a sector in the case of the two-directional system, which may introduce severe difficulties during the mechanical design of the stripping foil holder. Considering that these beams are of higher importance, the corresponding stripping foil position placed in the valley in the case of the unidirectional system is a big advantage. The uniqueness of the combined element may be a disadvantage of the unidirectional extraction. Since the combined magnetic element is not a standard element, special effort must be put into its design and manufacturing, and this will increase its cost.

\section{RESULTS}

As stated earlier, the stripping extraction system performance, as well as the system design parameters, depends on the key design parameter, $\theta_{e}$. During the system design process, the restrictions, requirements, and dependencies of the system performance on $\theta_{e}$ are used to determine the optimum value of $\theta_{e}$. In the previous section it was found that, for the VINCY Cyclotron, the optimal value of the key design parameter is $\theta_{e}=6^{\circ}$. The determined value is further used to define all the design parameters of the stripping extraction system: the operating area of the stripping foil, the placement, dimensions, and focusing and/or bending characteristics of the beam-guiding elements.

The operating area of the stripping foil is shown in Fig. 5. If the foil cannot be placed over a sector, due to difficulties related to the mechanical design of the foil

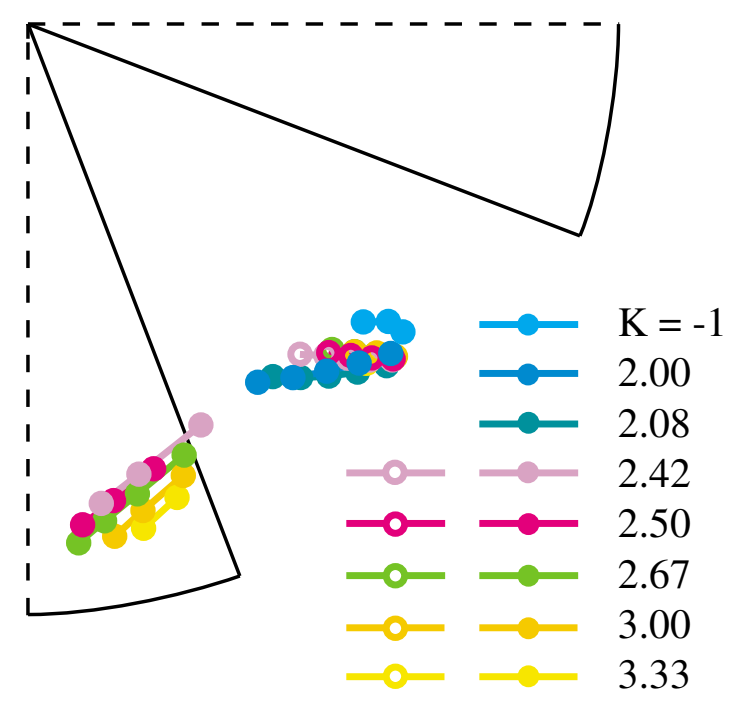

FIG. 5. (Color) Stripping foil area. The foil placements that bring the beams into the optimal exit point are shown. The foil positions that provide the best obtainable beam quality are marked with solid symbols. For those $K$ 's for which the optimal foil positions fall over the sector, the available backup positions in the valley are marked with open symbols. For $K=$ 2.5 and $K=2.42$ the optimal foil positions corresponding to the largest values of $R_{m}$ had to be disregarded, since the corresponding beam loops after the foil pass through the acceleration gap. 
positioning mechanism, the backup foil positions are available in the valley.

The horizontal layout of the chosen test beams and the beam-guiding elements is superimposed to the schematics of the VINCY Cyclotron in Fig. 6. The proposed unidirectional stripping extraction is more compact and less spacious compared to the two-directional design of the stripping extraction system [7-9].

The emittances and envelopes of the test beams with $K=-1,2,2.5$, and 3 are given in Figs. 7-10, respectively. To ease the comparison between the test beams, the figures have the same structure and the corresponding graphs have the same scale. The scale of the horizontal beam emittance matches the transport line acceptance. The scale of the vertical beam emittance is larger than the corresponding transport line acceptance to enable representation of the results obtained for the $\mathrm{H}_{2}{ }^{+}$ion beam. The minimal horizontal and vertical apertures of the combined and bending magnets used in these figures enable all simulated beams to pass through the magnets without collision and are $D_{h}^{\mathrm{CM}}=25 \mathrm{~cm}, D_{z}^{\mathrm{CM}}=5 \mathrm{~cm}$, $D_{h}^{\mathrm{BM}}=15 \mathrm{~cm}$, and $D_{z}^{\mathrm{BM}}=5 \mathrm{~cm}$, respectively.

Tables I and II summarize the values of the combined and bending magnet parameters used in simulations to guide the higher priority beams of the VINCY Cyclotron through the extraction line and to the beginning of the

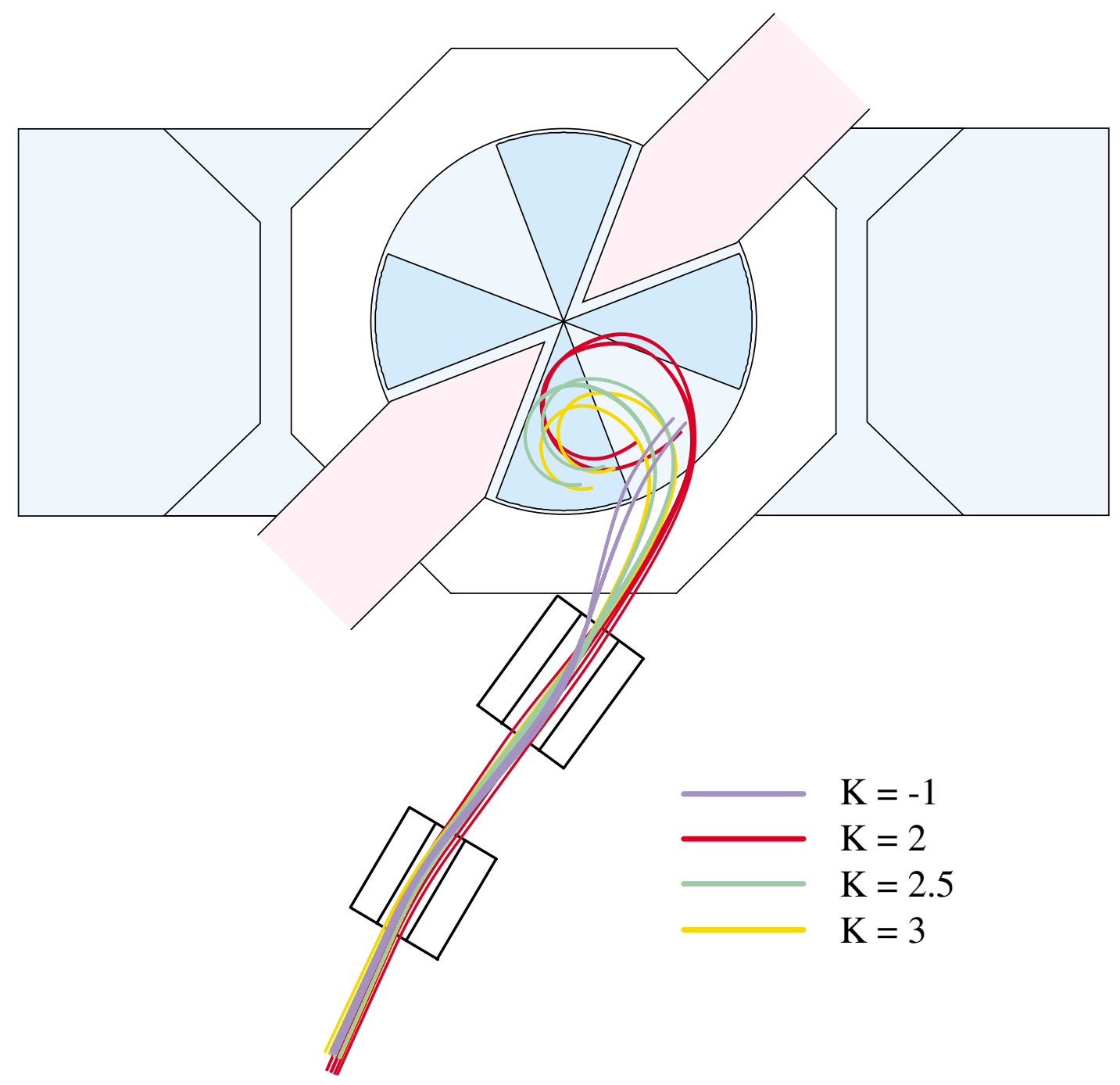

FIG. 6. (Color) Horizontal layout of test beams and beam-guiding elements. A test beam is represented by its central ion trajectory and horizontal envelope. For each $K$, the two test beams, corresponding to the minimum and the maximum $R_{m}$, are shown. The two beam-guiding elements of the extraction system, namely, the combined magnet, CM, and the bending magnet, BM, are represented by their placements and lengths, horizontal apertures, and estimated widths. The depicted magnet apertures represent $125 \%$ of the minimal apertures that enable all the test beams to pass through the magnets without collision. Note that the beams with $K=-1$ have the exit point $\theta_{e}=4^{\circ}$, not $\theta_{e}=6^{\circ}$. The trajectory offset of these highly bent beams enabled the decrease of the horizontal aperture of the combined magnet. In the case of the bending magnet, the horizontal aperture decrease was achieved by shifting the magnet center with respect to the beginning of the high-energy transport line. 

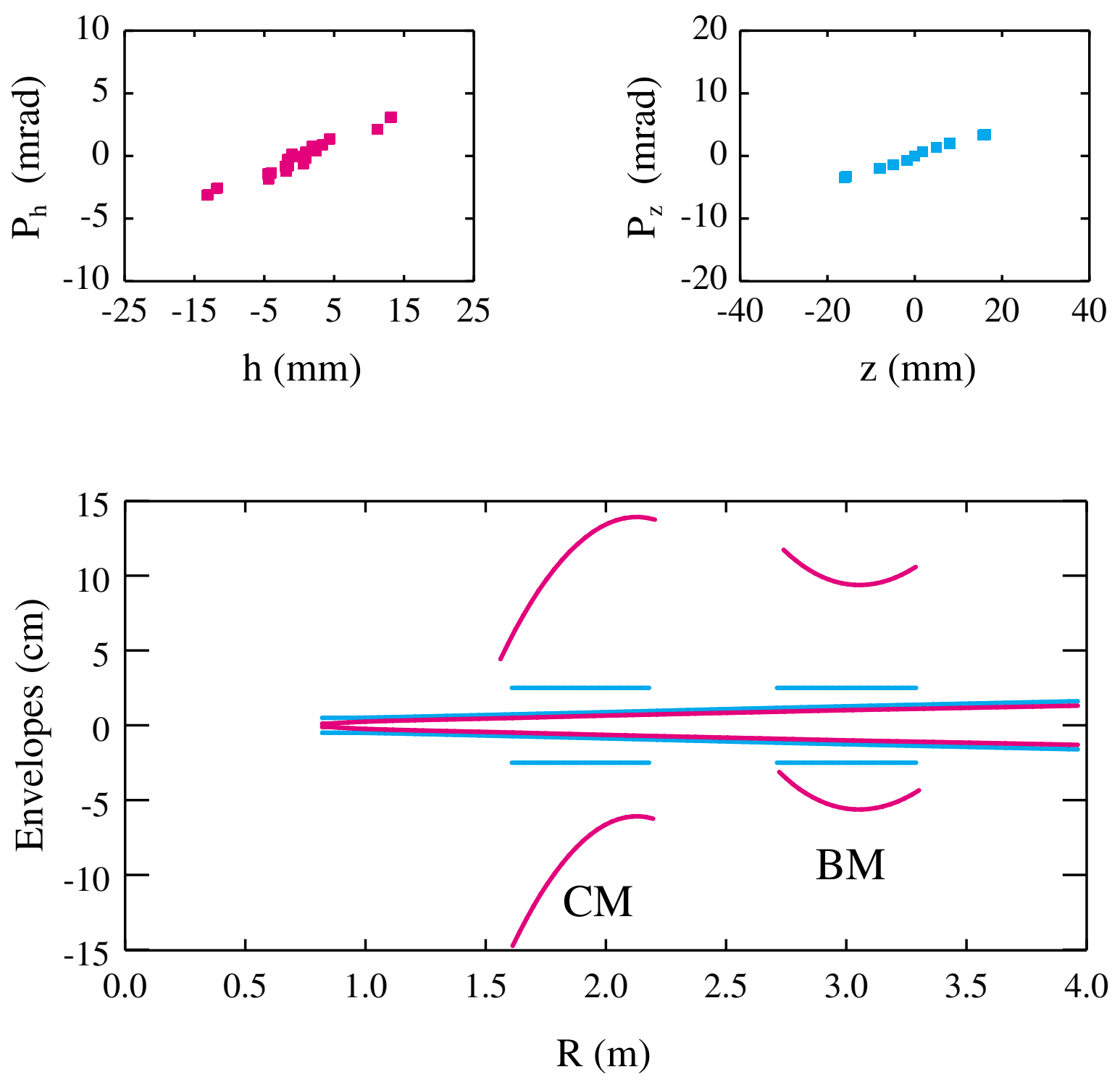

FIG. 7. (Color) Beam emittances and envelopes for $K=-1$. The beam emittances in the horizontal and vertical directions, represented in the upper left and upper right graphs, respectively, correspond to $R=4 \mathrm{~m}$, i.e., to the position along the high-energy transport line displaced $1 \mathrm{~m}$ from the transport line beginning. $h, P_{h}$ and $z, P_{z}$ are the spatial and momentum coordinates of a test ion with respect to the central test ion in the horizontal and vertical directions, respectively. The lower graph gives the radial dependence of the horizontal (magenta) and vertical (cyan) beam envelopes and apertures of the combined and bending magnets using the central ion trajectory as a referent zero axis. The central ion trajectory is confined to the median plane; therefore, for the vertical envelope and apertures the graph's zero axis corresponds to $z=0$, and the magnets' apertures are not distorted. The distortion of the horizontal magnet aperture is the consequence of the strong bending of the beam central trajectory inside the magnet. The presented emittances and envelopes correspond to the beam of protons obtained from $\mathrm{H}^{-}$ions accelerated to $65 \mathrm{MeV}^{-}$ The combined and bending magnet parameters used in the beam dynamics simulation are $B_{\mathrm{CM}}=0.56458 \mathrm{~T}, G=0 \mathrm{~T} / \mathrm{m}$, and $B_{\mathrm{BM}}=-0.47477 \mathrm{~T}$. The horizontal as well as the vertical emittance matches the required transport line acceptance. In the lower graph, the coordinate of the center of the combined magnet entrance is $\cong-5 \mathrm{~cm}$ and not $0 \mathrm{~cm}$ because the beam does not enter the combined magnet through its center positioned at $\theta_{e}=6^{\circ}$, but through the point with $\theta_{e}=4^{\circ}$.

high-energy transport line. Combining these data with those obtained for the beams with $K=2.5$ and $K=3$ revealed that the needed bending field range and the strongest focusing field of the combined magnet are $-0.70 \mathrm{~T} \leq B_{\mathrm{CM}} \leq 0.15 \mathrm{~T}$ and $G=1 \mathrm{~T} / \mathrm{m}$, respectively. The strongest needed field of the bending magnet is $B_{\mathrm{BM}}=-0.48 \mathrm{~T}$. When designing the combined and bending magnets, in order to avoid operation under limiting conditions, the operating ranges of the field and gradient values should be somewhat larger than those obtained as a result of beam dynamics simulations. 

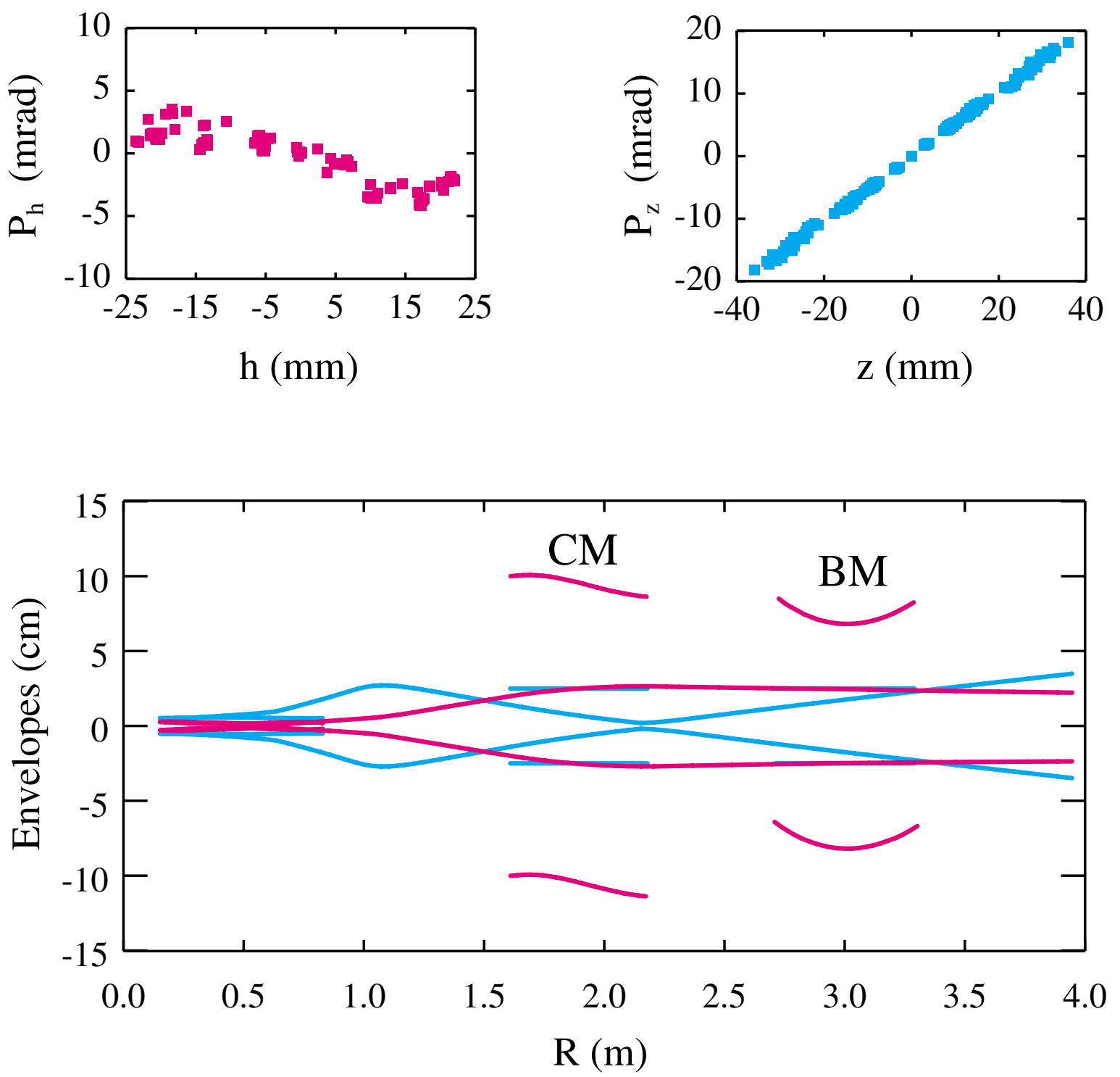

FIG. 8. (Color) Beam emittances and envelopes for $K=2$. The structure of the figure is the same as that of Fig. 7. The represented beam of protons was obtained from the $\mathrm{H}_{2}{ }^{+}$ion beam accelerated to $30 \mathrm{MeV}$ per nucleon. The combined and bending magnet parameters used in the beam dynamics simulation are $B_{\mathrm{CM}}=-0.12320 \mathrm{~T}, G=1 \mathrm{~T} / \mathrm{m}$, and $B_{\mathrm{BM}}=-0.29061 \mathrm{~T}$. The aperture of the combined magnet is only slightly distorted, so the beam central trajectory passes very close to the magnet axis. The horizontal emittance matches the transport line acceptance. The increase in $G$ cannot further improve the vertical emittance to match the transport line acceptance due to the small vertical beam size in the combined magnet.

The size of the combined magnet was estimated according to the $2 \mathrm{D}$ conceptual design of a similar magnet [10]. The designed magnet has the focusing field 3 times stronger and the bending field twice weaker than needed. The width of the combined magnet in Fig. 6 is $56 \mathrm{~cm}$ compared to the corresponding size of the designed magnet of $50 \mathrm{~cm}$. The vertical clearance of the vacuum chamber side is $34 \mathrm{~cm}$ and the height of the designed magnet is $23 \mathrm{~cm}$. Therefore, it is possible to shape the vacuum chamber side to accommodate the actual size of the combined magnet and its placement. Also, if needed, the length of the combined magnet can be somewhat increased (see Fig. 6).

\section{CONCLUSIONS}

Designing of a multipurpose machine is inevitably bound to a number of trade-offs. In the case of the stripping extraction system of the VINCY Cyclotron, the required beam diversity and quality are influenced and to some extent limited by the available space, the desired compactness of the extraction system, its complexity, and 

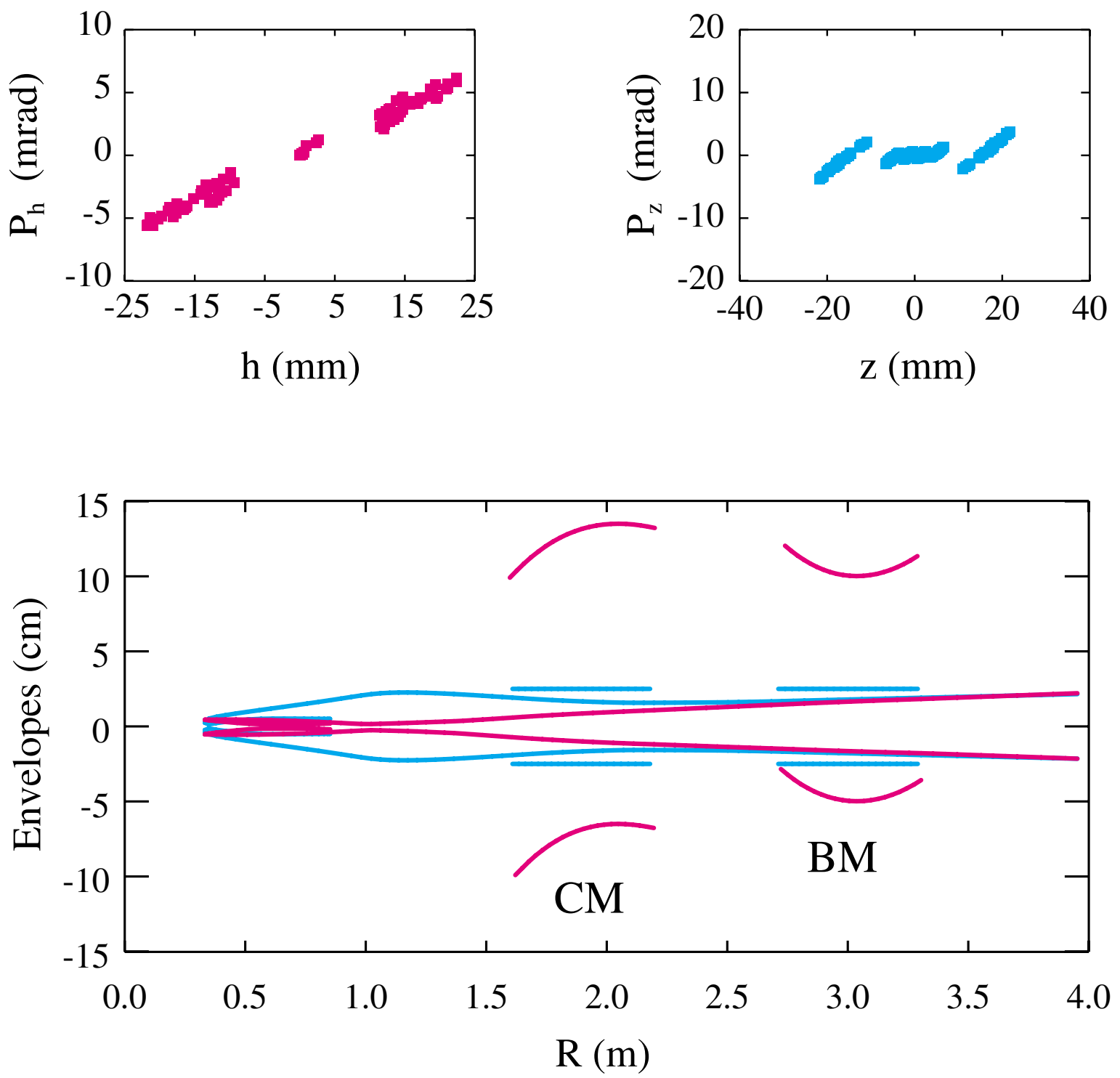

FIG. 9. (Color) Beam emittances and envelopes for $K=2.5$. The structure of the figure is the same as that of Fig. 7. Unlike the other examples, the mean equilibrium orbit radius in this case is not maximum, instead it is equal to $R_{m}=83.2 \mathrm{~cm}$. The beams extracted from equilibrium orbits with larger $R_{m}$ pass through the acceleration gap after the foil and had to be disregarded. The shown results correspond to the $\mathrm{Ar}^{15+}$ ion beam obtained from the $\mathrm{Ar}^{6+}$ ion beam with the energy of $2.9 \mathrm{MeV}$ per nucleon. The parameters of the beam-guiding elements are $B_{\mathrm{CM}}=0.07264 \mathrm{~T}, G=0.5 \mathrm{~T} / \mathrm{m}$, and $B_{\mathrm{BM}}=-0.25385 \mathrm{~T}$.

cost. A particular challenge is to design a system that successfully extracts both of the beams that have higher priority: the protons obtained from $\mathrm{H}^{-}$ion beams and the protons originating from $\mathrm{H}_{2}{ }^{+}$ion beams. Although it is a light ion beam, during extraction, the latter behaves as a heavy ion beam in terms of beam dynamics.

The dependencies of the beam direction on the beam exit point from the cyclotron reveal two possible concepts of the stripping extraction system. The two-directional system has two extraction branches that merge in the bending magnet at the beginning of the high-energy transport line. The beams accelerated as negative ions $\left(\mathrm{H}^{-},{ }^{2} \mathrm{H}^{-}\right)$are extracted without any size or direction, i.e., focusing or bending, corrections through the first extraction branch directly into the bending magnet at the beginning of the high-energy transport line. All other ion beams, particularly the protons originating from $\mathrm{H}_{2}{ }^{+}$ ions, require size corrections. Therefore, they are extracted into the focusing magnet placed at the beginning of the second extraction branch. The bending magnet positioned farther along this branch guides the beams into the bending magnet at the beginning of the highenergy transport line. The distance between the beam exits from the cyclotron corresponding to the two branches prevents the branches from being in the separate extraction tubes. Therefore, the two magnetic elements along the second branch are $\mathrm{C}$ shaped. 

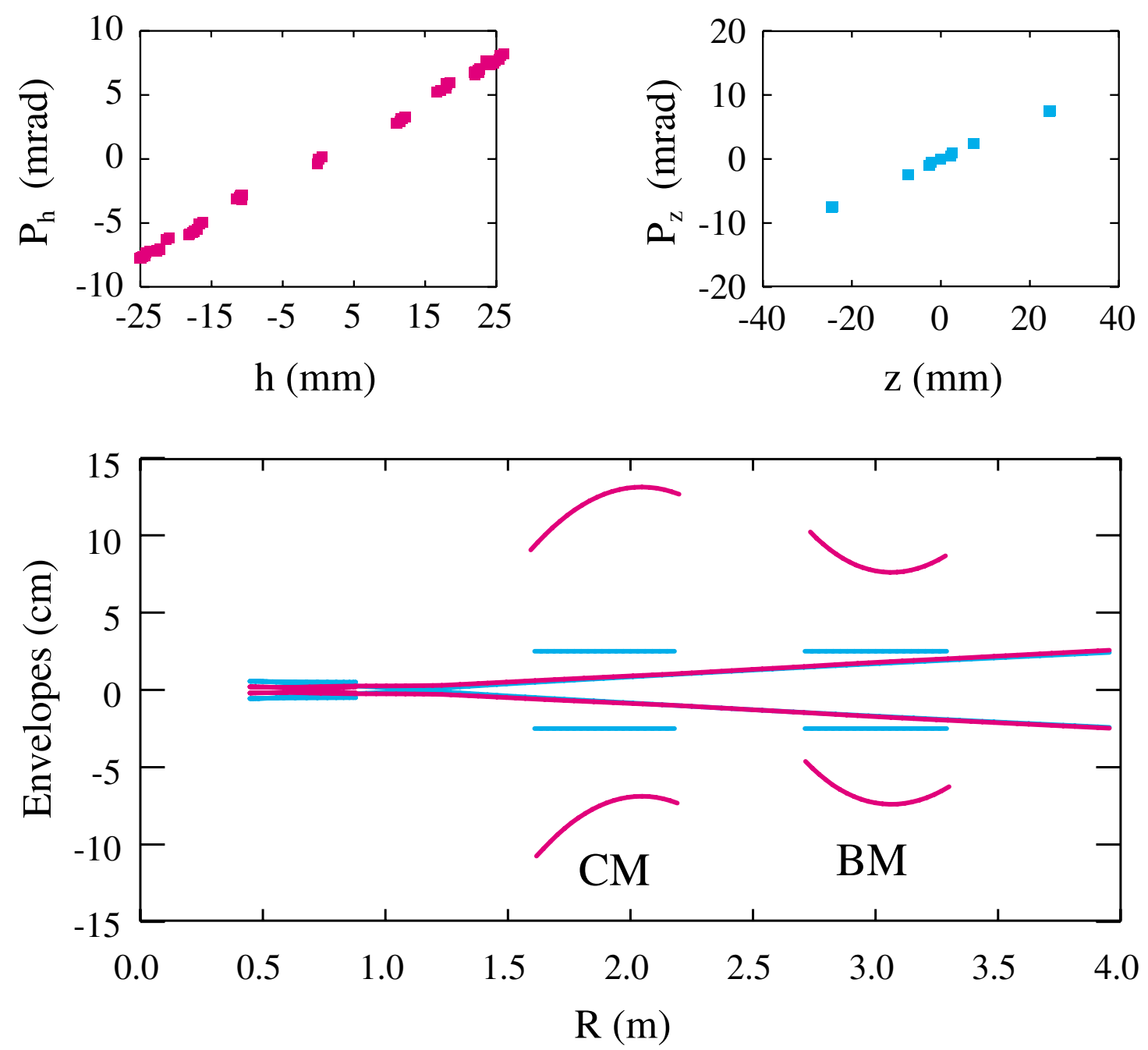

FIG. 10. (Color) Beam emittances and envelopes for $K=3$. The structure of the figure is the same as that of Fig. 7. The results correspond to the $\mathrm{Ne}^{9+}$ ion beam obtained after the interaction with the foil of the $\mathrm{Ne}^{3+}$ ion beam previously accelerated to the energy of $3.1 \mathrm{MeV}$ per nucleon. The corresponding parameters of the beam-guiding elements are $B_{\mathrm{CM}}=0.16474 \mathrm{~T}, G=0 \mathrm{~T} / \mathrm{m}$, and $B_{\mathrm{BM}}=-0.22878 \mathrm{~T}$. The horizontal size of the beam slightly exceeds the transport line acceptance; however, the adjustment of the horizontal width of the beam will enlarge the beam width in all of the remaining three emittance coordinates, namely, $P_{h}, z$, and $P_{z}$.

TABLE I. Focusing and bending fields for $\mathrm{H}^{-}$ion beams. The values of focusing and/or bending parameters of the combined and bending magnets used in beam dynamic simulations to properly guide beams of different energies are given. For the protons originating from $\mathrm{H}^{-}$ions, only the bending function of the combined magnet is used.

\begin{tabular}{clcc}
\hline \hline & \multicolumn{2}{l}{ Combined magnet } & Bending magnet \\
$T(\mathrm{MeV} / \mathrm{n})$ & $B_{\mathrm{CM}}(\mathrm{T})$ & $G(\mathrm{~T} / \mathrm{m})$ & $B_{\mathrm{BM}}(\mathrm{T})$ \\
\hline 65 & 0.56458 & 0 & -0.47477 \\
60 & 0.61798 & 0 & -0.47430 \\
55 & 0.70352 & 0 & -0.48116 \\
\hline \hline
\end{tabular}

TABLE II. Focusing and bending fields for $\mathrm{H}_{2}^{+}$ion beams. The focusing and/or bending properties of the combined and bending magnets corresponding to the ion beams of different energies are given. The proton beams originating from $\mathrm{H}_{2}{ }^{+}$ion beams are strongly focused and slightly bent in the combined magnet. For $T \leq 20 \mathrm{MeV}$ per nucleon a beam cannot be extracted without losses.

\begin{tabular}{cccc}
\hline \hline & \multicolumn{2}{c}{ Combined magnet } & Bending magnet \\
$T(\mathrm{MeV} / \mathrm{n})$ & $B_{\mathrm{CM}}(\mathrm{T})$ & $G(\mathrm{~T} / \mathrm{m})$ & $B_{\mathrm{BM}}(\mathrm{T})$ \\
\hline 30 & -0.12320 & 1.0 & -0.29061 \\
28 & -0.12707 & 1.0 & -0.28036 \\
26 & -0.13241 & 1.0 & -0.26899 \\
24 & -0.13890 & 1.0 & -0.25796 \\
22 & -0.14976 & 1.0 & -0.24181 \\
\hline \hline
\end{tabular}


The unidirectional system has a single extraction branch that connects the beam exit from the cyclotron and the high-energy transport line. Besides the bending magnet that is positioned at the beginning of the high-energy transport line, the unidirectional system has only one additional magnetic element-the combined magnet. The combined magnet has focusing as well as bending capabilities and is placed as close to the vacuum chamber as possible. The focusing function of the combined magnet is needed to correct the size of those beams that are accelerated as positive ions. The beams accelerated as negative ions do not need any size correction. However, their direction is significantly different from the extraction branch direction and requires strong and prompt bending. Capability of the combined magnet to provide focusing as well as bending of a beam improves the quality of the extracted beams. The bending function guides all beams close to the combined magnet axis and consequently focusing is more efficient. The combined magnet is a nonstandard element and therefore requires a special design.

Compared to the two-directional extraction, the unidirectional extraction has improved significantly the compactness of the stripping extraction system and the quality of the extracted beams. However, the combined magnetic element causes the unidirectional stripping extraction system to be more complex and likely more expensive. The design and development of the combined element is likely to be profitable, since such an element could become a standard component of an extraction system as well as of a transport line.

\section{ACKNOWLEDGMENTS}

The work was funded by the Ministry of Science, Technologies and Development of Serbia.
[1] N. Nešković, J. Ristić-Djurović, S. B. Vorojtsov, P. Belićev, I. A. Ivanenko, S. Ćirković, A. S. Vorojtsov, B. Bojović, A. Dobrosavljević, V. Vujović, J. J. Čomor, and S. B. Pajević, Nukleonika (to be published).

[2] S. Ćirković and J. L. Ristić-Djurović, in Proceedings of the 21st Summer School and International Symposium on the Physics of Ionized Gases (SPIG), Sokobanja, Yugoslavia, 2002, edited by M. K. Radović and M.S. Jovanović (University of Niš, Niš, 2002), p. 112.

[3] J. L. Ristić-Djurović, N. Nešković, and S. Ćirković, in Proceedings of the 6th International Computational Accelerator Physics Conference, Darmstadt, Germany, 2000, http://www.icap2000.de/

[4] S. Ćirković, J. Ristić-Djurović, and M. Milošević, in Proceedings of the 6th International Computational Accelerator Physics Conference, Darmstadt, Germany, 2000 (Ref. [3]).

[5] J.L. Ristić-Djurović, Phys. Rev. ST Accel. Beams 4, 123501 (2001).

[6] J. L. Ristić-Djurović and S. Ćirković, in Proceedings of the 21st Summer School and International Symposium on the Physics of Ionized Gases (SPIG), Sokobanja, Yugoslavia, 2002 (Ref. [2]), p. 108.

[7] O. N. Borisov, G. G. Gulbekyan, and J. Ristić-Djurović, in Proceedings of the 8th Information Meeting on the TESLA Accelerator Installation, Belgrade, Yugoslavia, 1996, edited by N. Nešković (Vinča Institute of Nuclear Sciences, Belgrade, 1999), p. 122.

[8] J. Ristić-Djurović and S. B. Vorozhtsov, in Proceedings of the 15th Information Meeting on the TESLA Accelerator Installation, Belgrade, Yugoslavia, 1998, edited by N. Nešković (Vinča Institute of Nuclear Sciences, Belgrade, 2000), p. 73.

[9] B. N. Gikal, O. N. Borisov, G. G. Gulbekyan, V. N. Melnikov, G. N. Ivanov, V.V. Bechterev, I.V. Kolesov, V. Vujović, J. Ristić-Djurović, N. Nešković, and Đ. Košutić, in Proceedings of the 15th Information Meeting on the TESLA Accelerator Installation, Belgrade, Yugoslavia, 1998 (Ref. [8]), p. 82.

[10] Ingvar Blomqvist, DANFYSIK (private communication). 